\title{
Decay suppression of spontaneous emission of a single emitter in a high- $Q$ cavity at exceptional points
}

\author{
M. Khanbekyan ${ }^{1,2, *}$ and J. Wiersig (1) ${ }^{1}$ \\ ${ }^{1}$ Institut für Physik, Otto-von-Guericke-Universität Magdeburg, Postfach 4120, D-39016 Magdeburg, Germany \\ ${ }^{2}$ Institut für Physik, Universität Rostock, Albert-Einstein-Straße 23, D-18059 Rostock, Germany
}

(Received 13 November 2019; accepted 29 May 2020; published 22 June 2020)

\begin{abstract}
The exceptional point (EP) is a non-Hermitian degeneracy of an open quantum system, where the behavior of the system is of a fundamental distinguishing nature. Within the framework of exact quantum electrodynamics in dispersing and absorbing media, we study the strong-coupling regime of a single emitter-high- $Q$ cavity system in the case where two cavity modes and the corresponding resonant frequencies coalesce at an EP. We demonstrate a substantial increase in the effective decay time of the spontaneous emission in the EP case. We show that this effect is directly related to the peculiar spectral response of the electromagnetic field at the EP. We apply the concept to the case of a microdisk cavity perturbed by nanoparticle scatterers, where the spontaneous emission decay suppression can be related to the parameters of the real system.
\end{abstract}

DOI: 10.1103/PhysRevResearch.2.023375

\section{INTRODUCTION}

Non-Hermitian physical systems have attracted much attention recently for their unconventional behavior around exceptional points (EPs), which are defined as spectral singularities at which two or more eigenvalues and their associated eigenvectors coalesce [1]. These singularities do not have equivalents in Hermitian systems, because the eigenvectors of non-Hermitian systems, which coalesce at the EP, are biorthogonal, in contrast to Hermitian systems, where the eigenvectors are orthogonal [2]. In particular, EPs have been studied in various physical contexts bringing new features to the overall response of optical systems [3], which lead to a number of intriguing phenomena such as loss-induced light transmission [4,5], enhanced sensing [6-8], and mode selection in lasers $[9,10]$. In practice, EPs and related effects have become experimentally accessible in many photonic systems [11].

In relation to the study of light-matter interaction, it has recently been shown that spontaneous emission of dipole emitters in the weak-coupling regime can be modified at an EP, leading to strong enhancement of the emission rate $[12,13]$. Generally, control of the spontaneous emission of fluorescent emitters near a resonant environment, which is typically characterized by the Purcell factor [14], attracts great interest for a wide range of applications, from sensing, where the modification of spectral properties of emitters is used to increase the detection sensitivity, to quantum information processing, where efficient single-photon sources are needed. The spontaneous emission rate enhancement is related to the linear response of a resonant system, e.g., an optical cavity, which

\footnotetext{
*khanbekyan@gmail.com

Published by the American Physical Society under the terms of the Creative Commons Attribution 4.0 International license. Further distribution of this work must maintain attribution to the author(s) and the published article's title, journal citation, and DOI.
}

in the case of an EP exhibits a narrowed, squared Lorentzian line shape, with a larger peak value in comparison to the single-resonance case. This effect makes it possible to modify the frequency response near the EP resonance [15-17].

In this paper, we exploit the modification of the response of a high- $Q$ cavity operating at an EP and study the spontaneous emission of a single emitter in the strong-coupling regime. In particular, within the frame of exact quantum electrodynamics (QED) we investigate the emission of the emitter embedded in a cavity supporting a second-order EP at a nearly resonant frequency. As we discuss below, the dynamics of the emitterfield interaction at an EP results from two features of the EP: the larger emission peak value at the EP in comparison to the single-mode case and the narrower emission peak of a squared Lorentzian line shape compared to that of a standard Lorentzian spectrum.

\section{GENERAL THEORY}

We consider a single emitter (position $\mathbf{r}_{A}$ ) that interacts with the electromagnetic field in the presence of a dispersing and absorbing dielectric medium and assume that only a single transition $\left(|1\rangle \leftrightarrow|2\rangle\right.$; frequency $\omega_{21}$, dipole-moment $\mathbf{d}_{21}$ ) is quasiresonantly coupled to a narrow-band cavityassisted electromagnetic field. Applying the multipolarcoupling scheme in the electric dipole approximation, we write the Hamiltonian that governs the temporal evolution of the overall system, which consists of the electromagnetic field, the dielectric medium (including the dissipative degrees of freedom), and the emitter coupled to the field, in the form of (for details, see Appendix A and Refs. [18-20])

$$
\begin{aligned}
\hat{H}= & \int d^{3} r \int_{0}^{\infty} d \omega \hbar \omega \hat{\mathbf{f}}^{\dagger}(\mathbf{r}, \omega) \cdot \hat{\mathbf{f}}(\mathbf{r}, \omega)+\hbar \omega_{21} \hat{S}_{22} \\
& -\left[i \sqrt{\frac{\hbar}{\pi \varepsilon_{0}}} \int_{0}^{\infty} d \omega \frac{\omega^{2}}{c^{2}} \int d^{3} r \sqrt{\operatorname{Im} \varepsilon(\mathbf{r}, \omega)}\right. \\
& \left.\times \mathbf{d}_{21} \cdot \mathbf{G}\left(\mathbf{r}_{A}, \mathbf{r}, \omega\right) \cdot \hat{\mathbf{f}}(\mathbf{r}, \omega) \hat{S}_{21}+\text { H.c. }\right] .
\end{aligned}
$$


In this equation, the first term is the Hamiltonian of the field-medium system, where the fundamental bosonic fields $\hat{\mathbf{f}}(\mathbf{r}, \omega)$ and $\hat{\mathbf{f}}^{\dagger}(\mathbf{r}, \omega)$ play the role of the canonically conjugate system variables. The second term is the Hamiltonian of the emitter, where the $\hat{S}_{l l^{\prime}}$ are the flip operators, $\hat{S}_{l l^{\prime}}=|l\rangle\left\langle l^{\prime}\right|$, corresponding to the $|l\rangle \leftrightarrow\left|l^{\prime}\right\rangle$ transition, with $|l\rangle$ being the energy eigenstate of the emitter. Finally, the last term is the emitter-field coupling energy. In the above, the classical Green tensor $\mathbf{G}\left(\mathbf{r}, \mathbf{r}^{\prime}, \omega\right)$ defines the structure of the electromagnetic field in the presence of the dielectric bodies described by the spatially varying, frequency-dependent permittivity $\varepsilon(\mathbf{r}, \omega)$. In particular, the Green tensor determines the spectral response of the resonator cavity.

In what follows we assume that the emitter is initially (at time $t=0$ ) prepared in the upper state $|2\rangle$ and the cavity and electromagnetic field system is prepared in the ground state $|\{0\}\rangle$, defined by $\hat{\mathbf{f}}(\mathbf{r}, \omega)|\{0\}\rangle=0$. Then we may approximately span the Hilbert space of the whole system by the single-excitation states and expand the state vector of the overall system at later times $t(t \geqslant 0)$ as

$$
\begin{aligned}
|\psi(t)\rangle= & C_{2}(t) e^{-i \omega_{21} t}|\{0\}\rangle|2\rangle \\
& +\int d^{3} r \int_{0}^{\infty} d \omega e^{-i \omega t} \mathbf{C}_{1}(\mathbf{r}, \omega, t) \cdot \hat{\mathbf{f}}^{\dagger}(\mathbf{r}, \omega)|\{0\}\rangle|1\rangle .
\end{aligned}
$$

It is not difficult to show that the Schrödinger equation for $|\psi(t)\rangle$ leads to the following integrodifferential equation for $C_{2}(t)$ (see Ref. [21]):

$$
\dot{C}_{2}=\int_{0}^{t} d t^{\prime} K\left(t-t^{\prime}\right) C_{2}\left(t^{\prime}\right),
$$

with the initial condition $C_{2}(0)=1$. In the above, the kernel function $K(t)$ reads

$$
\begin{aligned}
K(t)= & -\frac{1}{\pi \hbar \varepsilon_{0}} \int_{0}^{\infty} d \omega \frac{\omega^{2}}{c^{2}} e^{-i\left(\omega-\omega_{21}\right) t} \\
& \times \mathbf{d}_{21} \cdot \operatorname{Im} \mathrm{G}\left(\mathbf{r}_{A}, \mathbf{r}_{A}, \omega\right) \cdot \mathbf{d}_{21}^{*} .
\end{aligned}
$$

\section{SPONTANEOUS EMISSION AT AN EP}

Let us assume that any expression $s(\omega)$ that includes the Green tensor can be presented as

$$
s(\omega)=\frac{S(\omega)}{D(\omega)},
$$

where the function $D(\omega)$ describes the resonant structure of the Green tensor and $S(\omega)$ is analytic in the lower half-plane. Let $s(t)$ be a function of time whose Fourier transform is given by

$$
s(\omega)=\int d t e^{i \omega t} s(t)
$$

with

$$
s(t)=\int \frac{d \omega}{2 \pi} e^{-i \omega t} \frac{S(\omega)}{D(\omega)} .
$$

In the case where the cavity features nondegenerate high$Q$ modes, the excitation spectrum effectively turns into a quasidiscrete set of resonant frequencies $\Omega_{l}=\omega_{l}-i \Gamma_{l} / 2$ according to the zeros of the function $D(\omega)$. Then, from Eqs. (6) and (7) we find

$$
s^{\mathrm{ND}}(\omega)=\sum_{l=1}^{N} \frac{S\left(\Omega_{l}\right)}{\omega-\Omega_{l}},
$$

which represents a sum of Lorentzian profiles.

In the case where the cavity is operating at a second-order $\mathrm{EP}$, that is, when two modes coalesce, forming an EP of complex frequency, $\Omega_{k}=\omega_{k}-i \Gamma_{k} / 2$, a second-order pole emerges in the Green tensor [22]. For the sake of simplicity, we assume that the emitter transition frequency $\omega_{21}$ is near the frequency $\omega_{k}$ and the influence of other modes is weak. Then, integrating Eq. (7) in the case where $s(\omega)$ features a pole of second order, from Eq. (6) we obtain

$$
s^{\mathrm{EP}}(\omega)=\frac{\alpha_{k}}{\left(\omega-\Omega_{k}\right)^{2}}+\frac{\beta_{k}}{\omega-\Omega_{k}},
$$

with $\alpha_{k}=S\left(\Omega_{k}\right)$ and $\beta_{k}=\left.[d S(\omega) / d \omega]\right|_{\omega=\Omega_{k}}$. Note that for $\omega$ close to the resonance frequency $\omega_{k}$,

$$
\operatorname{Im} s^{\mathrm{EP}}(\omega)=-\frac{\Gamma_{k}^{2}}{4} \frac{\operatorname{Im} \alpha_{k}}{\left|\omega-\Omega_{k}\right|^{4}}-\frac{\Gamma_{k}}{2} \frac{\operatorname{Re} \beta_{k}}{\left|\omega-\Omega_{k}\right|^{2}}
$$

approximately holds. Equation (10) reveals that in the case of a second-order EP the cavity spectral response features, in addition to the conventional linear Lorentzian profile, a square Lorentzian term associated with the singular spectral point.

To illustrate the effect induced by the squared Lorentzian term we insert Eq. (4) together with Eq. (10) into Eq. (3) and assume $\operatorname{Im} \alpha_{k}, \operatorname{Re} \beta_{k}<0$. Then we compare the results of the probability $\left|C_{2}(t)\right|^{2}$ and the spectral mode function of the outgoing field (for details of calculations, see Ref. [21]) for three cases. In the first case, we assume that only one mode is nearly resonant to the emitter transition. Then, applying Eq. (8) to the $\omega$-dependent function under the integral in Eq. (4), and assuming that only the $k$ th mode is quasiresonantly coupled to the emitter at the coupling rate $R_{k}$, we can solve the integrodifferential equation in Eq. (3). As Fig. 1(a) reveals, the amplitude of the upper level $\left|C_{2}(t)\right|^{2}$ reflects the emitter-field interaction, where the oscillations decay due to the finite lifetime of the cavity mode. From the right plot in Fig. 1(a) we can see that the spectral mode function features Rabi splitting of the strong emitter-field coupling. In the second case, we assume that two orthogonal modes with the same $\Omega_{k}$ are quasiresonantly coupled to the emitter at the coupling rate $R_{k}$ and apply Eq. (8) now with two modes. Then we can see from Fig. 1(b) that the spectral mode functions for both modes are identical and feature Rabi splitting, which is larger in comparison to that in the singlemode case.

Finally, to study the influence of the modified frequency response in the case of an EP, we consider the third case, the EP case, Eq. (9), where we first assume $\left|\operatorname{Im} \alpha_{k}\right| \gg \Gamma_{k}\left|\operatorname{Re} \beta_{k}\right|$, that is, the first term in Eq. (10) is much larger than the second one. In this case, the dynamics of the system is determined by the square Lorentzian line shape with an enhanced emission rate and reduced line width. As we see in the right plot in Fig. 1(c), the spectral mode function in this case again features Rabi splitting of the strong emitter-field coupling, and the Rabi splitting is nearly the same as in the single-mode case. 

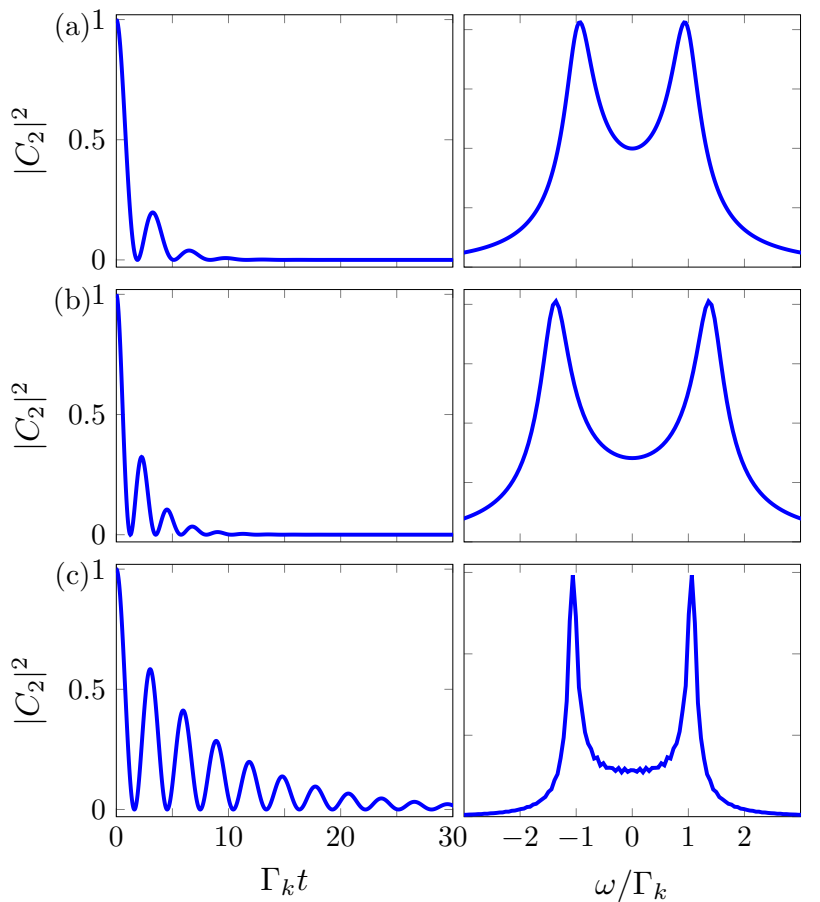

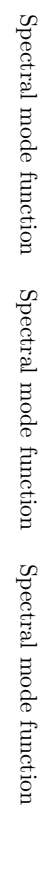

FIG. 1. Amplitude $\left|C_{2}(t)\right|^{2}$ and spectral mode function of the outgoing field for $\omega_{21}=\omega_{k}=10000 \Gamma_{k}$ and $R_{k}=2 \Gamma_{k}$ : (a) in the single-mode case, (b) in the two-mode case, and (c) in the EP case.

Most importantly, the comparison of the plots of the left column in Fig. 1 reveals that the effective decay in the EP case is smaller than in the single-mode and double-mode cases. Similarly, as the right column in Fig. 1 reveals, the line width of the Rabi peaks is smaller in the case of the EP in comparison with the single-mode and double-mode cases. Effectively, the single excitation decay rate is much lower in the EP case, causing longer oscillations between the emitter and the cavity field.

In general, both the linear and the square Lorentzian terms may contribute to the Green tensor. In particular, from Eq. (10) it can be seen that the contributions of the linear and square Lorentzian terms in the kernel function $K(t)$ are proportional to $\operatorname{Re} \beta_{k}$ and $\operatorname{Im} \alpha_{k}$, respectively. Thus, by increasing the proportion $\left|\operatorname{Im} \alpha_{k} /\left(\Gamma_{k} \operatorname{Re} \beta_{k}\right)\right|$ we can make the EP contribution dominant. In this way, as we can see from the plots of the amplitude of the upper level $\left|C_{2}(t)\right|^{2}$ in Fig. 2, the effective decay time can be substantially increased.

To explain the above-mentioned results, we note that the presence of the EP leads to a higher emission rate in comparison to the single-mode case. At the same time, a large emission at the EP is compensated by narrowing of the mode line width. The consequence is comparable values of the Rabi frequency in the single-mode and the EP cases but an effectively lower decay rate in the EP case.

As an example of an optical cavity that can operate at an EP we consider a microdisk cavity perturbed by a non-Hermitian backscattering [23]. The clockwise (CW) and counterclockwise $(\mathrm{CCW})$ propagating modes can be coupled by two nanoparticle scatterers introducing asymmetric backscattering, which may lead to non-Hermitian degeneracy with coalescing frequencies, decay rates, and eigenmodes. For

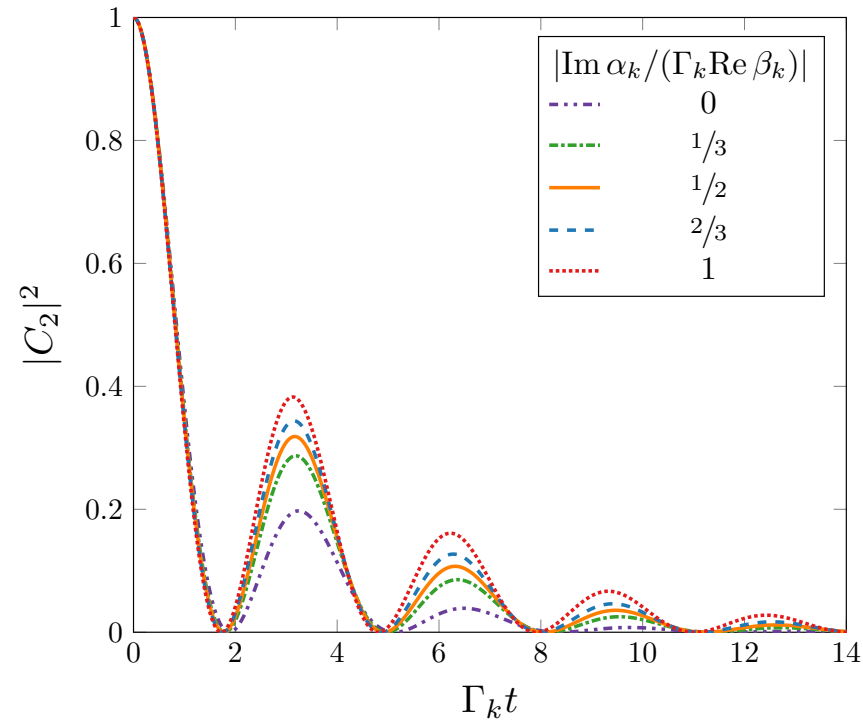

FIG. 2. Amplitude $\left|C_{2}(t)\right|^{2}$ for various $\left|\operatorname{Im} \alpha_{k} /\left(\Gamma_{k} \operatorname{Re} \beta_{k}\right)\right|$. Parameters are the same as in Fig. 1.

the description of the EP, we use a two-mode approximation, assuming that the nanoparticles introduce a small perturbation that couples only the modes within a given degenerate mode pair of the isolated microdisk. We consider CW and CCW modes in the angular momentum representation with the given mode azimuthal number $m$, where the wave function inside the cavity can be given by means of cylindrical harmonics,

$$
\begin{aligned}
\psi(\rho, \phi)= & \psi_{+}(t) J_{m}\left(n \omega_{k} \rho / c\right) e^{i m \phi} \\
& +(-1)^{m} \psi_{-}(t) J_{m}\left(n \omega_{k} \rho / c\right) e^{-i m \phi},
\end{aligned}
$$

with $J_{m}$ being the $m$ th-order Bessel function of the first kind and $n \equiv n(\rho)$ the piecewise constant refractive index of the microdisk cavity. Then, in the slowly varying envelope approximation the Maxwell equations reduce to the following Schrödinger-type equation for the wave function $\boldsymbol{\psi} \equiv \boldsymbol{\psi}(t)=$ $\left(\psi_{+}(t), \psi_{-}(t)\right)^{\mathrm{T}}$, with $\mathrm{T}$ denoting the transposed vector (see Ref. [23], for details):

$$
i \frac{d}{d t} \boldsymbol{\psi}=\mathcal{H} \boldsymbol{\psi}
$$

where the effective Hamiltonian reads

$$
\mathcal{H}=\left(\begin{array}{ll}
\Omega & A \\
B & \Omega
\end{array}\right) .
$$

In the above, the complex-valued parameters $A$ and $B$, describing the scattering from the $\mathrm{CW}$ to the $\mathrm{CCW}$ and from the CCW to the CW traveling waves, depend on the size, refractive index, and location of the scatterers. The right eigenvectors of the Hamiltonian Eq. (13) corresponding to the solution with outgoing boundary condition read

$$
\boldsymbol{\psi}_{ \pm}^{\mathrm{R}}=\left(\begin{array}{c}
\sqrt{A} \\
\pm \sqrt{B}
\end{array}\right)
$$

with eigenvalues $\Omega_{ \pm}=\Omega \pm \sqrt{A B}$. Here, we are interested in the particular case where by appropriate choice of the parameters of the scatterers, backscattering in a sole direction 
vanishes with $B=0$. Then an EP is realized, where the eigenfrequencies $\Omega_{ \pm}$and eigenvectors $\psi_{ \pm}^{\mathrm{R}}$ coalesce, and the remaining eigenstate is a pure $\mathrm{CCW}$ wave with the eigenvalue $\Omega_{k}$ and the right eigenvector

$$
\boldsymbol{\psi}_{\mathrm{EP}}^{\mathrm{R}}=\left(\begin{array}{l}
1 \\
0
\end{array}\right),
$$

which, together with the left eigenvector $\left(\psi_{\mathrm{EP}}^{\mathrm{L}}\right)^{\mathrm{T}}=\left(\begin{array}{ll}0 & 1\end{array}\right)$, satisfies the self-orthogonality condition $\left(\boldsymbol{\psi}_{\mathrm{EP}}^{\mathrm{L}}\right)^{\mathrm{T}} \cdot \boldsymbol{\psi}_{\mathrm{EP}}^{\mathrm{R}}=0$. Note that considering the Hamiltonian $\mathcal{H}$ as parameter dependent from $B$, in the neighborhood of the EP (small $|B|$ ), the Hamiltonian has two nearly parallel eigenvectors $\psi_{ \pm}^{R}$ with nearly degenerate eigenvalues $\Omega_{ \pm}$. Then the Green tensor $\mathrm{G}\left(\mathbf{r}, \mathbf{r}^{\prime}, \omega\right)$ can be approximately given by a non-Hermitian quasinormal expansion [24] which, close to the resonant frequency $\omega_{k}$ (in the nondispersive case [25]), can be presented by means of the wave functions defined in the basis of $\mathrm{CW}$ and CCW traveling waves (Appendix B; see, also, Ref. [1]):

$$
\stackrel{\leftrightarrow}{G}(\omega)=\frac{1}{\omega-\Omega_{+}} \frac{\boldsymbol{\psi}_{+}^{\mathrm{R}}\left(\boldsymbol{\psi}_{+}^{\mathrm{L}}\right)^{\mathrm{T}}}{\left(\boldsymbol{\psi}_{+}^{\mathrm{L}}\right)^{\mathrm{T}} \cdot \boldsymbol{\psi}_{+}^{\mathrm{R}}}+\frac{1}{\omega-\Omega_{-}} \frac{\boldsymbol{\psi}_{-}^{\mathrm{R}}\left(\boldsymbol{\psi}_{-}^{\mathrm{L}}\right)^{\mathrm{T}}}{\left(\boldsymbol{\psi}_{-}^{L}\right)^{\mathrm{T}} \cdot \boldsymbol{\psi}_{-}^{\mathrm{R}}} .
$$

Further, using perturbative expansion for eigenvalues $\Omega_{ \pm}$and eigenvectors $\boldsymbol{\psi}_{ \pm}^{\mathrm{R}, \mathrm{L}}$ around the EP (see, e.g., Ref. [26] for the use of the Newton-Puiseux expansion in the vicinity of EPs) and taking the limit of $B \rightarrow 0$, we find

$$
\begin{aligned}
\stackrel{\leftrightarrow}{G}_{\mathrm{EP}}(\omega)= & \frac{1}{\left(\omega-\Omega_{k}\right)^{2}} \frac{\boldsymbol{\psi}_{\mathrm{EP}}^{\mathrm{R}}\left(\boldsymbol{\psi}_{\mathrm{EP}}^{\mathrm{L}}\right)^{\mathrm{T}}}{\left(\boldsymbol{\psi}_{\mathrm{EP}}^{L}\right)^{\mathrm{T}} \cdot \mathbf{j}_{\mathrm{EP}}^{\mathrm{R}}} \\
& +\frac{1}{\omega-\Omega_{k}} \frac{\boldsymbol{\psi}_{\mathrm{EP}}^{\mathrm{R}}\left(\mathbf{j}_{\mathrm{EP}}^{\mathrm{L}}\right)^{\mathrm{T}}+\mathbf{j}_{\mathrm{EP}}^{\mathrm{R}}\left(\boldsymbol{\psi}_{\mathrm{EP}}^{\mathrm{L}}\right)^{\mathrm{T}}}{\left(\boldsymbol{\psi}_{\mathrm{EP}}^{L}\right)^{\mathrm{T}} \cdot \mathbf{j}_{\mathrm{EP}}^{\mathrm{R}}}
\end{aligned}
$$

where the Jordan vectors $\mathbf{j}_{\mathrm{EP}}^{\mathrm{R}, \mathrm{L}}$ are defined by the relations

$$
\begin{gathered}
\mathcal{H} \mathbf{j}_{\mathrm{EP}}^{\mathrm{R}}=\Omega_{k} \mathbf{j}_{\mathrm{EP}}^{\mathrm{R}}+\boldsymbol{\psi}_{\mathrm{EP}}^{\mathrm{R}}, \\
\left(\mathbf{j}_{\mathrm{EP}}^{\mathrm{L}}\right)^{\mathrm{T}} \mathcal{H}=\Omega_{k}\left(\mathbf{j}_{\mathrm{EP}}^{\mathrm{L}}\right)^{\mathrm{T}}+\left(\boldsymbol{\psi}_{\mathrm{EP}}^{\mathrm{L}}\right)^{\mathrm{T}},
\end{gathered}
$$

together with the normalization conditions $\left(\mathbf{j}_{\mathrm{EP}}^{\mathrm{L}}\right)^{\mathrm{T}} \cdot \mathbf{j}_{\mathrm{EP}}^{\mathrm{R}}=0$ and $\left(\boldsymbol{\psi}_{\mathrm{EP}}^{\mathrm{L}}\right)^{\mathrm{T}} \cdot \mathbf{j}_{\mathrm{EP}}^{\mathrm{R}}=A^{-1}$. Then, using Eqs. (13), (15), (18), and (19) from Eq. (17), we obtain

$$
\stackrel{\leftrightarrow}{G}_{\mathrm{EP}}(\omega)=\left(\begin{array}{cc}
\frac{1}{\omega-\Omega_{k}} & \frac{A}{\left(\omega-\Omega_{k}\right)^{2}} \\
0 & \frac{1}{\omega-\Omega_{k}}
\end{array}\right) .
$$

As we can see, the structure of the Green tensor reflects the asymmetric character of the effective non-Hermitian Hamiltonian, Eq. (13), with $B=0$. In particular, a consequence of this structure is the asymmetric backscattering at an EP, e.g., in the case of a cavity with two scatterers $[26,27]$ or weakly (and asymmetrically) deformed cavities [28-30], where the reflection coefficient exhibits a resonant peak in one propagation direction but vanishes in the other.

Most importantly, Eq. (20) reveals that by means of adjustment of the parameter of the asymmetric backscattering $A$ the square Lorentzian term can be made prevailing in comparison to the standard Lorentzian term. In practice, the parameters of the system can be controlled by adjusting the sizes, the size relation, and the relative phase angle between the scatterers. Note that for passive cavities the inequality $|A| \leqslant \Gamma_{k}$ holds ([7]; but also see [31]). Thus, in the case of a strong interaction with the emitter, effective decay times of the spontaneous emission can be substantially increased by adjusting the parameter $\operatorname{Im} A$ from 0 (linear Lorentzian regime) to larger than $\Gamma_{k}$ (square Lorentzian regime); cf. Fig. 2 with $\left|\operatorname{Im} \alpha_{k} /\left(\Gamma_{k} \operatorname{Re} \beta_{k}\right)\right|=1$.

\section{SUMMARY AND DISCUSSION}

In summary, starting with macroscopic QED theory in dispersing and absorbing media, we have presented an exact description of the resonant interaction of a two-level emitter in a high- $Q$ cavity in the case where two cavity modes form an EP. The quantum theory allows the study of the properties of spontaneous emission of the single emitter. The spectral response of the resonator cavity is described by a square Lorentzian, in contrast to a Lorentzian function in the single-mode case. The consequence is much longer effective decay times in the EP case in comparison to the single-mode case. High effective $Q$ values of the cavity mode allow the reaching of high emission rates of the emitter into the cavity mode robust against dephasing effects, which can be used, e.g., to achieve high indistinguishability of single-photon generation-a building block for many scalable quantum information technologies, where indistinguishability is required to realize a photonphoton interaction achieved by means of quantum interference effects [32]. Moreover, the usage of EP resonances in cavity QED may open possibilities for new practical approaches of nonlinear optical interaction at the single-photon level [33]. We would like to emphasize that the theory presented in this work has a general validity, and the results suggest the possibility of experimental observation of the emission decay suppression in a wide range of photonic systems, which can operate at an EP. Obviously, the theory can be applied over a wide range of experimentally relevant parameters of different non-Hermitian systems in the regime of strong coupling. In particular, the theory can be applied to optical waveguides with strong evanescent coupling to a molecular dipole [34] or coupled whispering-gallery mode microresonators [4,5], two-dimensional photonic crystal slabs [35], and microring resonators with an embedded angular structure [36] or two nanotip scatterers [27], where a strong coupling regime with a single semiconductor quantum dot [37-39] can be achieved. Further decay suppression is possible if the system operates at a higher-order EP [40,41], in which case the Green tensor features higher-order Lorentzian functions. Another question to ask is the sensitivity of the results with respect to a small change in parameters that moves the system away from the EP. For example, as shown in Ref. [2], the eigenfunctions that coalesce at an EP become almost orthogonal to each other by a weak disturbance that steers the system away from the EP.

\section{ACKNOWLEDGMENT}

The authors are grateful to J. Kullig for helpful discussions. 


\section{APPENDIX A: BASICS OF THE MACROSCOPIC QED}

The theory of macroscopic QED is an extension of vacuum QED that describes the quantized electromagnetic field in the presence of macroscopic dielectric bodies. Local, inhomogeneous, linear dielectric bodies can be characterized by a spatially varying permittivity $\varepsilon(\mathbf{r}, \omega)$, which is a complex function of the frequency. The Hamiltonian of the electromagnetic field and the dielectric medium is given by $[18,19]$

$$
\hat{H}_{\text {field }}=\int d^{3} r \int_{0}^{\infty} d \omega \hbar \omega \hat{\mathbf{f}}^{\dagger}(\mathbf{r}, \omega) \cdot \hat{\mathbf{f}}(\mathbf{r}, \omega),
$$

which integrates the medium-modified creation and annihilation operators of the medium-field system over the entire space in position and frequency. The medium-field bosonic operators satisfy the commutation relations

$$
\begin{gathered}
{\left[\hat{\mathbf{f}}(\mathbf{r}, \omega), \hat{\mathbf{f}}^{\dagger}\left(\mathbf{r}^{\prime}, \omega^{\prime}\right)\right]=\delta\left(\omega-\omega^{\prime}\right) \delta\left(\mathbf{r}-\mathbf{r}^{\prime}\right),} \\
{\left[\hat{\mathbf{f}}(\mathbf{r}, \omega), \hat{\mathbf{f}}\left(\mathbf{r}^{\prime}, \omega^{\prime}\right)\right]=\mathbf{0} .}
\end{gathered}
$$

The expression for the medium-assisted electric field is defined by the field operators and the dyadic Green tensor,

$$
\begin{aligned}
\hat{\mathbf{E}}(\mathbf{r})= & \int_{0}^{\infty} d \omega\left[\hat{\mathbf{E}}(\mathbf{r}, \omega)+\hat{\mathbf{E}}^{\dagger}(\mathbf{r}, \omega)\right] \\
= & i \sqrt{\frac{\hbar}{\varepsilon_{0} \pi}} \int_{0}^{\infty} d \omega \frac{\omega^{2}}{c^{2}} \int d^{3} r^{\prime} \sqrt{\operatorname{Im} \varepsilon\left(\mathbf{r}^{\prime}, \omega\right)} \\
& \times \mathbf{G}\left(\mathbf{r}, \mathbf{r}^{\prime}, \omega\right) \cdot \hat{\mathbf{f}}\left(\mathbf{r}^{\prime}, \omega\right)+\text { H.c. }
\end{aligned}
$$

where the Green tensor solves the Helmholtz equation for the electric field resulting from the Maxwell equations as

$\nabla \times \nabla \times G\left(\mathbf{r}, \mathbf{r}^{\prime}, \omega\right)-\frac{\omega^{2}}{c^{2}} \varepsilon(\mathbf{r}, \omega) \mathrm{G}\left(\mathbf{r}, \mathbf{r}^{\prime}, \omega\right)=\delta^{(3)}\left(\mathbf{r}-\mathbf{r}^{\prime}\right)$,

together with the boundary condition at infinity, $\mathrm{G}\left(\mathbf{r}, \mathbf{r}^{\prime}, \omega\right) \rightarrow 0$ if $\left|\mathbf{r}-\mathbf{r}^{\prime}\right| \rightarrow \infty$. The emitter Hamiltonian can be given by

$$
\hat{H}_{\mathrm{A}}=\sum_{m} \hbar \omega_{m m} \hat{S}_{m m}
$$

with $\hat{S}_{m n}$ being the flip operators,

$$
\hat{S}_{m n}=|m\rangle\langle n|,
$$

where $|m\rangle$ are the energy eigenstates of the emitter. Finally, the emitter-field coupling energy

$$
\hat{H}_{\text {int }}=-\hat{\mathbf{d}} \cdot \hat{\mathbf{E}}\left(\mathbf{r}_{A}\right)
$$

contains the electric dipole-moment operator of the emitter, which can be represented by means of the flip operators as

$$
\hat{\mathbf{d}}=\sum_{m n} \mathbf{d}_{m n} \hat{S}_{m n}
$$

with $\mathbf{d}_{m n}=\langle m|\hat{\mathbf{d}}| n\rangle$. The Hamiltonian of the overall system consists of the medium field, emitter, and interaction parts:

$$
\hat{H}=\hat{H}_{\text {field }}+\hat{H}_{\mathrm{A}}+\hat{H}_{\mathrm{int}} \text {. }
$$

Further, we assume that only a single emitter transition $|1\rangle \leftrightarrow$ $|2\rangle$ is involved, with the emitter eigenenergy $\omega_{11}=0$. Then $\omega_{22}=\omega_{21}$, where $\omega_{21}$ is the single transition frequency. Thus, inserting Eqs. (A1), (A6), and (A8) together with Eq. (A4) into Eq. (A10) we obtain the total Hamiltonian, Eq. (1).

\section{APPENDIX B: MODAL EXPANSION OF THE GREEN TENSOR}

In general, the spectral representation of a non-Hermitian Hamiltonian can be given in terms of the left and right eigenvectors as

$$
\mathcal{H}=\sum_{l} \Omega_{l} \frac{\boldsymbol{\psi}_{l}^{\mathrm{R}}\left(\boldsymbol{\psi}_{l}^{\mathrm{L}}\right)^{\mathrm{T}}}{\left(\boldsymbol{\psi}_{l}^{\mathrm{L}}\right)^{\mathrm{T}} \cdot \boldsymbol{\psi}_{l}^{\mathrm{R}}}
$$

Resonances of the Hamiltonian in the Schrödinger-type equation, Eq. (12), can be defined in the Fourier space as the poles of the Green tensor or the resolvent $(\omega \mathcal{I}-\mathcal{H})^{-1}$. Similarly to Eq. (B1) the mode expansion of the Green function reads

$$
\stackrel{\leftrightarrow}{G}(\omega)=\sum_{l} \frac{1}{\omega-\Omega_{l}} \frac{\boldsymbol{\psi}_{l}^{\mathrm{R}}\left(\boldsymbol{\psi}_{l}^{\mathrm{L}}\right)^{\mathrm{T}}}{\left(\boldsymbol{\psi}_{l}^{L}\right)^{\mathrm{T}} \cdot \boldsymbol{\psi}_{l}^{\mathrm{R}}} .
$$

In the vicinity of an EP the terms of the expansion with vanishing denominators are dominant. Thus, we obtain Eq. (16).
[1] N. Moiseyev, Non-Hermitian Quantum Mechanics (Cambridge University Press, Cambridge, UK, 2011).

[2] H. Eleuch and I. Rotter, Phys. Rev. A 93, 042116 (2016).

[3] M.-A. Miri and A. Alù, Science 363, 42 (2019).

[4] B. Peng, Ş. K. Özdemir, F. Lei, F. Monifi, M. Gianfreda, G. L. Long, S. Fan, F. Nori, C. M. Bender, and L. Yang, Nat. Phys. 10, 394 (2014).

[5] L. Chang, X. Jiang, S. Hua, C. Yang, J. Wen, L. Jiang, G. Li, G. Wang, and M. Xiao, Nat. Photon. 8, 524 (2014).

[6] J. Wiersig, Phys. Rev. Lett. 112, 203901 (2014).

[7] J. Wiersig, Phys. Rev. A 93, 033809 (2016).

[8] W. Chen, Ş. K. Özdemir, G. Zhao, J. Wiersig, and L. Yang, Nature 548, 192 (2017).

[9] L. Feng, Z. J. Wong, R.-M. Ma, Y. Wang, and X. Zhang, Science 346, 972 (2014)
[10] H. Hodaei, M.-A. Miri, M. Heinrich, D. N Christodoulides, and M. Khajavikhan, Science 346, 975 (2014).

[11] H. Cao and J. Wiersig, Rev. Mod. Phys. 87, 61 (2015).

[12] A. Pick, B. Zhen, O. D. Miller, C. W. Hsu, F. Hernandez, A. W. Rodriguez, M. Soljačić, and S. G. Johnson, Opt. Express 25 , 12325 (2017).

[13] Z. Lin, A. Pick, M. Lončar, and A. W. Rodriguez, Phys. Rev. Lett. 117, 107402 (2016).

[14] E. M. Purcell, Phys. Rev. 69, 37 (1946).

[15] A. Pick, Z. Lin, W. Jin, and A. W. Rodriguez, Phys. Rev. B 96, 224303 (2017).

[16] S. Sunada, Phys. Rev. A 97, 043804 (2018).

[17] G. Yoo, H.-S. Sim, and H. Schomerus, Phys. Rev. A 84, 063833 (2011). 
[18] L. Knöll, S. Scheel, and D.-G. Welsch, Coherence and Statistics of Photons and Atoms (Wiley, New York, 2001), p. 1; arXiv:quant-ph/0006121.

[19] W. Vogel and D.-G. Welsch, Quantum Optics, 3rd ed. (WileyVCH, Weinheim, 2006).

[20] M. Khanbekyan, D.-G. Welsch, C. Di Fidio, and W. Vogel, Phys. Rev. A 78, 013822 (2008).

[21] M. Khanbekyan, Phys. Rev. A 97, 023809 (2018).

[22] W. D. Heiss and G. Wunner, Eur. Phys. J. D 68, 284 (2014).

[23] J. Wiersig, Phys. Rev. A 84, 063828 (2011).

[24] W. D. Heiss, Int. J. Theor. Phys. 54, 3954 (2015).

[25] The exact calculations of the Green tensor for dispersive and absorbing cylindrical cavity including scattering particles are beyond the scope of the present paper.

[26] U. Günther, I. Rotter, and B. F. Samsonov, J. Phys. A: Math. Theor. 40, 8815 (2007).

[27] B. Peng, Ş. K. Özdemir, M. Liertzer, W. Chen, J. Kramer, H. Yilmaz, J. Wiersig, S. Rotter, and L. Yang, Proc. Natl. Acad. Sci. USA 113, 6845 (2016).

[28] J. Wiersig, S. W. Kim, and M. Hentschel, Phys. Rev. A 78, 053809 (2008).

[29] J. Wiersig, A. Eberspächer, J.-B. Shim, J.-W. Ryu, S. Shinohara, M. Hentschel, and H. Schomerus, Phys. Rev. A 84, 023845 (2011).

[30] J. Kullig and J. Wiersig, Phys. Rev. A 100, 043837 (2019).
[31] J. Wiersig, Phys. Rev. Research 1, 033182 (2019).

[32] T. Northup and R. Blatt, Nat. Photon. 8, 356 (2014).

[33] K. M. Birnbaum, A. Boca, R. Miller, A. D. Boozer, T. E. Northup, and H. J. Kimble, Nature 436, 87 (2005).

[34] J. Hwang and E. Hinds, New J. Phys. 13, 085009 (2011).

[35] B. Zhen, C. W. Hsu, Y. Igarashi, L. Lu, I. Kaminer, A. Pick, S.. Chua, J. D. Joannopoulos, and M. Soljačić, Nature 525, 354 (2015).

[36] P. Miao, Z. Zhang, J. Sun, W. Walasik, S. Longhi, N. M. Litchinitser, and L. Feng, Science 353, 464 (2016).

[37] J. P. Reithmaier, G. Sek, A. Löffler, C. Hofmann, S. Kuhn, S. Reitzenstein, L. V. Keldysh, V. D. Kulakovskii, T. L. Reinecke, and A. Forchel, Nature 432, 197 (2004).

[38] E. Peter, P. Senellart, D. Martrou, A. Lemaître, J. Hours, J. M. Gérard, and J. Bloch, Phys. Rev. Lett. 95, 067401 (2005).

[39] K. Hennessy, A. Badolato, M. Winger, D. Gerace, M. Atatüre, S. Gulde, S. Fält, E. L. Hu, and A. Imamoğlu, Nature 445, 896 (2007).

[40] H. Hodaei, A. U. Hassan, S. Wittek, H. Garcia-Gracia, R. ElGanainy, D. N. Christodoulides, and M. Khajavikhan, Nature 548, 187 (2017).

[41] S. Wang, B. Hou, W. Lu, Y. Chen, Z. Q. Zhang, and C. T. Chan, Nat. Commun. 10, 832 (2019). 